\title{
Review Article \\ Colored HOMFLY Polynomials as Multiple Sums over Paths or Standard Young Tableaux
}

\author{
A. Anokhina, ${ }^{1,2}$ A. Mironov, ${ }^{2,3}$ A. Morozov, ${ }^{2}$ and And. Morozov ${ }^{2,4}$ \\ ${ }^{1}$ MIPT, 9 Institutsky Per., Dolgoprudny 141700, Russia \\ ${ }^{2}$ ITEP, 25 Bol. Cheremushkinskaya, Moscow 117259, Russia \\ ${ }^{3}$ Lebedev Physics Institute, 53 Leninsky Pr., Moscow 119991, Russia \\ ${ }^{4}$ Moscow State University, GSP-1, Moscow 119991, Russia \\ Correspondence should be addressed to A. Mironov; mironov@lpi.ru
}

Received 22 May 2013; Accepted 7 September 2013

Academic Editor: Kadayam S. Viswanathan

Copyright (C) 2013 A. Anokhina et al. This is an open access article distributed under the Creative Commons Attribution License, which permits unrestricted use, distribution, and reproduction in any medium, provided the original work is properly cited.

If a knot is represented by an $m$-strand braid, then HOMFLY polynomial in representation $R$ is a sum over characters in all representations $Q \in R^{\otimes m}$. Coefficients in this sum are traces of products of quantum $\widehat{\mathscr{R}}$-matrices along the braid, but these matrices act in the space of intertwiners, and their size is equal to the multiplicity $M_{R Q}$ of $Q$ in $R^{\otimes m}$. If $R$ is the fundamental representation $R=[1]=\square$, then $M_{\square Q}$ is equal to the number of paths in representation graph, which lead from the fundamental vertex $\square$ to the vertex $Q$. In the basis of paths the entries of the $m-1$ relevant $\widehat{\mathscr{R}}$-matrices are associated with the pairs of paths and are nonvanishing only when the two paths either coincide or differ by at most one vertex, as a corollary $\widehat{\mathscr{R}}$-matrices consist of just $1 \times 1$ and $2 \times 2$ blocks, given by very simple explicit expressions. If cabling method is used to color the knot with the representation $R$, then the braid has as many as $m|R|$ strands; $Q$ have a bigger size $m|R|$, but only paths passing through the vertex $R$ are included into the sums over paths which define the products and traces of the $m|R|-1$ relevant $\widehat{\mathscr{R}}$-matrices. In the case of $S U(N)$, this path sum formula can also be interpreted as a multiple sum over the standard Young tableaux. By now it provides the most effective way for evaluation of the colored HOMFLY polynomials, conventional or extended, for arbitrary braids.

\section{Introduction}

Knot polynomials are currently among the central objects of interest in quantum field theory; they are exactly at the border between the known and unknown. The knot polynomials can be defined as Wilson loop averages:

$$
H_{R}^{\mathscr{K} C \mathscr{M}}(q \mid G)=\left\langle\operatorname{tr}_{R} P \exp \left(\oint_{\mathscr{K}} \mathscr{A}\right)\right\rangle_{C S},
$$

that is, generic gauge-invariant observables in the simplest version of the 3-dimensional Yang-Mills theory and the topological Chern-Simons model [1] with the action:

$$
\frac{k}{4 \pi} \int_{\mathscr{M}} \operatorname{tr}\left(\mathscr{A} d \mathscr{A}+\frac{2}{3} \mathscr{A}^{3}\right),
$$

and they depend on a closed contour $\mathscr{K}$ in a three dimensional manifold $\mathscr{M}$, on the representation $R$ of the gauge group $G=S U(N)$ and on the coupling constant $q=$ $e^{2 \pi i /(k+N)}$. Since the theory is topological, the dependence is actually only on the topological class of $\mathscr{K}$; that is, the contour can be considered as a knot. For the simply connected space $\mathscr{M}=R^{3}$ or $S^{3}$ the average $H(q \mid G)$ is actually a polynomial in $q$ and $A=q^{N}$, hence, the name "knot polynomial."

The study of knot polynomials in topology goes back to [2-7], and they were put into the context of quantum field theory in the seminal works by Schwarz [8] and Witten [9], further developed in [10-13]. Since the theory is topological, there are no dynamical phenomena like confinement, instead a close relation exists to the 2-dimensional conformal theories $[9,14-18]$, very much in the spirit of AdS/CFT correspondence [19-21]. In this way the knot polynomials are related to the most difficult part of conformal field theory, to modular transformations, which through the AGT relations 
[22-32] are connected to the $S$-duality between the $\mathcal{N}=2$ supersymmetric Yang-Mills theories [33-44]. This makes the study of knot polynomials the next task after the structure of conformal blocks themselves more or less understood in terms of the Dotsenko-Fateev matrix models [45-55] and other similar representations [56, 57].

An additional interest is induced by existence of nontrivial deformations of the knot polynomials: to superpolynomials [58-66] and refined Chern-Simons theory [67, 68] (see also [69]), to extended knot polynomials [70], which puts them into the class of $\tau$-function like objects, and so forth. At least naively $[71,72]$, they belong to the family of Hurwitz partition functions [73-76], more general than the conventional $\mathrm{KP} /$ Toda $\tau$-functions, probably related to the generalized $\tau$ functions of [77-80].

However, for any kind of generic investigation and application of knot polynomials, they should be first effectively calculated and represented in a theoretically appealing form, allowing evaluation of these polynomials for particular knots and representations. There are different competitive approaches to do this, for example, [81-84]. The goal of this letter is to summarize the results of our method [70, 8587], which provides a complete, nice, and practically efficient solution to this problem.

\section{HOMFLY Polynomials via Quantum $\mathscr{R}$-Matrices}

The method may begin with choosing the temporal gauge $A_{0}=0$ [88-90] in Chern-Simons theory; then the theory becomes quadratic with the ultralocal propagator $\theta(t) \delta(\vec{x})$. Then the original knot in 3-dimensions is substituted by a 2dimensional knot diagram (a 4-valent oriented graph), and the Wilson average reduces to a $q$-graded trace of the product of quantum $\mathscr{R}$-matrices, standing at the vertices of the graph [10-12].

It is most convenient to choose the knot diagram in the form of a closure of a braid. If the braid has $m$ strands, then the product involves $m-1$ different $\mathscr{R}$-matrices: $\mathscr{R}_{(i)}$ stands at the intersection of strands $i$ and $i+1$, and $i=1, \ldots, m-1$. For instance, for the 3 -strand braid one has

$$
H_{R}^{\left(a_{1}, b_{1}\left|a_{2}, b_{2}\right|, \ldots\right)}=\operatorname{tr}_{R^{\otimes 3}}^{\mathrm{grad}} \mathscr{R}_{(1)}^{a_{1}} \mathscr{R}_{(2)}^{b_{1}} \mathscr{R}_{(1)}^{a_{2}} \mathscr{R}_{(2)}^{b_{2}} \cdots
$$

In the pattern picture $a_{1}=0, b_{1}=-2, a_{2}=2, b_{2}=-1$, and $a_{3}=3$ (see Figure 1).

Similarly, for arbitrary $m$

$$
\begin{aligned}
& H_{R}^{\left(a_{11}, \ldots, a_{1, m-1}\left|a_{21}, \ldots, a_{2, m-1}\right|, \ldots\right)} \\
& \quad=\operatorname{tr}_{R^{\otimes m}}^{\mathrm{grad}} \mathscr{R}_{(1)}^{a_{11}} \cdots \mathscr{R}_{(m-1)}^{a_{1, m-1}} \mathscr{R}_{(1)}^{a_{21}} \cdots \mathscr{R}_{(m-1)}^{a_{2, m-1}} \cdots .
\end{aligned}
$$

The trace here is defined with additionally inserted element $q^{R^{\otimes m}}$ so that

$$
\chi_{Q}=\operatorname{tr}_{Q}^{\operatorname{grad}} I=\operatorname{dim}_{q}^{G}(Q)
$$

are the quantum dimensions of the representation $R$ (the characters of the group $G$ at the special values $p_{k}=\left(A^{k}-\right.$ $\left.\left.A^{-k}\right) /\left(q^{k}-q^{-k}\right)\right)$. In this formula the $\mathscr{R}$ matrices are of the huge size $\operatorname{dim}(R)^{2} \times \operatorname{dim}(R)^{2}$ and this expression can seem absolutely hopeless to evaluate for generic group $G$ and representation $R$.

However, things are actually much simpler. The product $R^{\otimes m}$ can be expanded into a sum of irreducible representations, generically, with nontrivial multiplicities. The crucial observation is that $\mathscr{R}_{(i)}$ act as unity in each irreducible representation, so that the matrices in (4) can be reduced to $\widehat{\mathscr{R}}_{(i)}$ of a much smaller size, equal to just the multiplicities $M_{R Q}$ of $Q$ in $R^{\otimes m}[70,85]$ :

$$
\begin{gathered}
H_{R}^{\mathscr{K}}(G)=\mathfrak{N}^{w(\mathscr{K})} \sum_{\mathrm{Q} \in R^{\otimes m}} C_{R Q}^{\mathscr{K}} \chi_{\mathrm{Q}}(G) \\
C_{R Q}^{\left(a_{11} \cdots a_{1, m-1} \mid \cdots\right)}=\operatorname{tr}_{M_{R Q}} \widehat{\mathscr{R}}_{(1)}^{a_{11}} \ldots \widehat{\mathscr{R}}_{(m-1)}^{a_{1, m-1}} \widehat{\mathscr{R}}_{(1)}^{a_{11}} \cdots \widehat{\mathscr{R}}_{(m-1)}^{a_{2, m-1}} \cdots
\end{gathered}
$$

Here $\mathfrak{N}$ is a normalization factor which emerges due to our choice of nonstandard normalization of $\mathscr{R}$-matrices and $w(\mathscr{K})$ is the writhe number. The standard normalization of $\mathscr{R}$-matrix in the vertical framing is restored with the factor $q^{-2 \varkappa_{R}}$, where $\varkappa_{R}=\sum_{i, j \in R}(j-i)$ and the sum runs over the coordinates $(i, j)$ of the boxes in the Young diagram that corresponds to the representation $R$. In order to restore the topological invariance, one has to change framing with a factor of $A^{-|R|} q^{-2 \varkappa_{R}}$ which totally gives $\mathfrak{N}=A^{-|R|} q^{-4 \varkappa_{R}}$.

What is important, in formula (7) the knot and group dependencies are separated and one can consider $H_{R}$ as a function of $A=q^{N}$ rather than $N$; the parameter $N$ enters only the quantum dimensions $\chi_{\mathrm{Q}}$ and can be easily substituted by $A$. Moreover, this formula actually introduces the extended HOMFLY polynomial $H_{R}\{p\}$, if $\chi_{\mathrm{Q}}$ are interpreted as characters, which are functions of infinitely many timevariables $\left\{p_{k}\right\}$ instead of $N$ or $A[70,85]$. The topological invariance is, however, lost beyond the topological locus $p_{k}=$ $p_{k}^{*}=\left(A^{k}-A^{-k}\right) /\left(q^{k}-q^{-k}\right)$.

To deal with this formula one needs an explicit expression for the $\widehat{\mathscr{R}}$-matrices; see $[85,91,92]$. Those papers contain many various observations about the structure of $\widehat{\mathscr{R}}$-matrices, and they were used to calculate many nontrivial knot polynomials; still a complete description is not yet found on that way, except for the fundamental representation case of $R=[1]=\square$, fully described in [86], and for the (anti)symmetric representation case [91-93] (some results in this latter case are also reproduced by alternative methods [94-100]).

It is therefore natural to attack the case of arbitrary $R$ with the help of the cabling approach [101] and apply the results of [86]. This is successfully done in $[87,102]$; this letter is a short summary of $[86,87]$, purified from all the details and extensive list of examples evaluated there. 


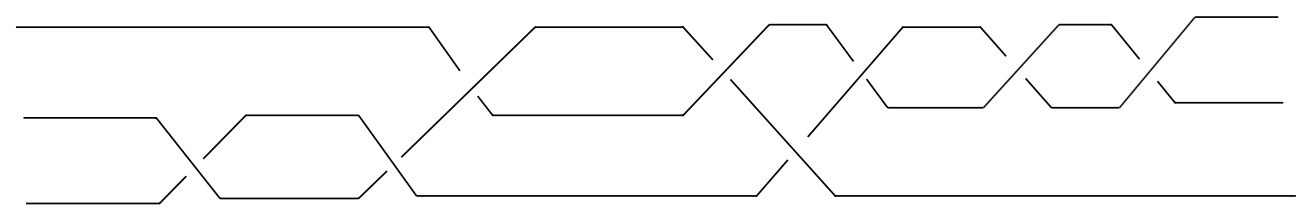

Figure 1

Note also that here we restrict our discussion to the knots only. The links can be dealt with similarly; however, they require some technical complications; thus, for the sake of brevity, we skip this extension (see details in [87]).

\section{3. $\widehat{\mathscr{R}}$-Matrices via Paths in the Representation Graph}

Since the cabling reduces the problem from $R$ to the case of the fundamental representation, the results of [86] are directly applicable [87] and we begin from repeating them in a concise and pictorial form (see Figure 2).

Implicit in [86] is representation of the coefficients $C_{\square \mathrm{Q}}$ in the form of a sum over paths in the representation graph [87]. The first four levels of the representation graph of [85] are shown in Figure 2; in an obvious way it describes the multiplication of fundamental representations [1]. The multiplicity $M_{\square Q}$ of the representation $Q$ in $\square^{\otimes|Q|}$ is obviously equal to the number of directed paths in the representation graph, connecting $\square$ and $Q$. More generally, $M_{R Q}$ is equal to the number of directed paths between $R$ and $Q$. The matrices $\widehat{\mathscr{R}}_{(i)}, i=1, \ldots, m-1$ can be represented in the basis of paths between $Q$ and $\square$ and according to $[86,87]$ they have extremely simple form in this basis.

First of all, with each index $i$ of the matrix $\widehat{\mathscr{R}}_{(i)}$ one associates a level $i$ in the graph. A given path $\mathscr{P}$ is passing through exactly one vertex $P_{i}$ at level $i$ and through some two adjacent vertices $P_{i-1}$ and $P_{i+1}$ at levels $i-1$ and $i+1$. The structure of the representation graph is such that these $P_{i-1}$ and $P_{i+1}$ are connected either by a single two-segment path (singlet) (then it is a fragment of our $P$ ) or by two such paths (doublet), the segments of our path $\mathscr{P}$ and another path $\mathscr{P}^{\prime}$. We call the transformations $\mathscr{P} \leftrightarrow \mathscr{P}^{\prime}$ a flip (see Figure 3 ).

In the former case (singlet), our path $\mathscr{P}$ provides a diagonal element in $\widehat{\mathscr{R}}_{(i)}$ and it is equal to either $q$ or $-1 / q$. In the language of Young diagrams the singlet appears when the two boxes added to the diagram $P_{i-1}$ in order to form $P_{i+1}$ lie either in the same row, then we put $q$ at the diagonal of $\widehat{\mathscr{R}}_{i}$, or in the same column, then we put $-1 / q$ (see Figure 4 ).

In the latter case (the doublet) (see Figure 5), the two boxes are neither in the same row nor in the same column, and the two paths $\mathscr{P}$ and $\mathscr{P}^{\prime}$ form a $2 \times 2$ block in $\widehat{\mathscr{R}}_{(i)}$. This block is described as follows. First, that of the paths $\mathscr{P}$ and $\mathscr{P}^{\prime}$ which lies to the left of the other, corresponds to the left column and to the first row of the $2 \times 2$ block. Second, the Young diagrams $P_{i+1}$ is obtained by adding two boxes to the diagram $P_{i-1}$, and the two paths correspond to doing this in two different orders, thus providing at the intermediate stage the two adjacent vertices $P_{i}$ and $P_{i}^{\prime}$. The two added boxes are

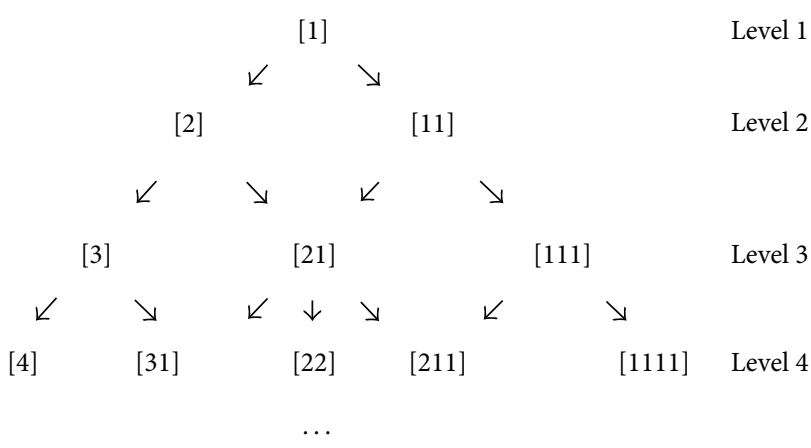

Figure 2

connected by a hook in the Young diagram, which has length $n$ (measured between the centers of the two boxes). Then the $2 \times 2$ block is equal to

$$
\begin{gathered}
\left(\begin{array}{cc}
-q^{-n} c_{n} & s_{n} \\
s_{n} & q^{n} c_{n}
\end{array}\right), \quad c_{n}=\frac{1}{[n]_{q}}=\frac{q-q^{-1}}{q^{n}-q^{-n}}, \\
s_{n}=\sqrt{1-c_{n}^{2}}=\frac{\sqrt{[n-1]_{q}[n+1]_{q}}}{[n]_{q}} .
\end{gathered}
$$

Figure 5 shows that for $P_{i-1}=$ [5521], $P_{i+1}=$ [6522], $P_{i}=$ [6521], and $P_{i}^{\prime}=$ [5522], the parameter $n=7$.

This provides a complete and very explicit description of the matrices $\widehat{\mathscr{R}}_{(i)}, i=1, \ldots, m-1$, which appear under the trace in the expression for $C_{\square Q}$. Note that in this form these matrices depend on $Q$. This description can seem rather lengthy and tricky, but in practice it is very algorithmic, easily programmable, and very effective for practical calculations [87].

The paths from $\square$ to $Q$ in the representation graph are labeled by the standard Young tableaux, that is, the Young diagram $Q$ with the numbers $1, \ldots,|Q|$ assigned to its boxes in such a way that the emerging sequences are increasing along each row and each column. The number in a given box is just the number of steps, at which the box was attached to the diagram when moving from $\square$ to $Q$. The number of paths is therefore equal to the well-known number of the standard Young tableaux,

$$
M_{\square \mathrm{Q}}=\frac{|\mathrm{Q}| !}{\prod_{\text {boxes } \in \mathrm{Q}} L(\text { box })},
$$

where $L$ (box) is the length of the hook in $Q$ associated to the box.

Thus the central formula (7) can be considered as a multiple sum over the standard Young tableaux of $Q$, and 


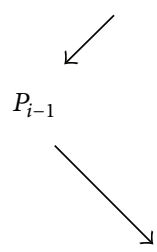

$P_{i}$

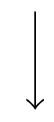

$P_{i+1}$

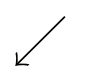

Example of a singlet

(no other path between $P_{i-1}$ and $P_{1+1}$ )

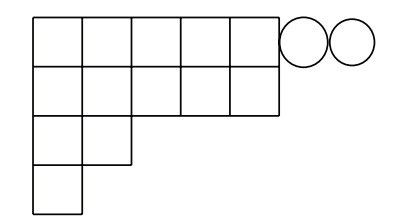

$[5521] \longrightarrow[6521] \longrightarrow[7521]$

The diagonal entry for the path $\mathscr{P}$ is $q$

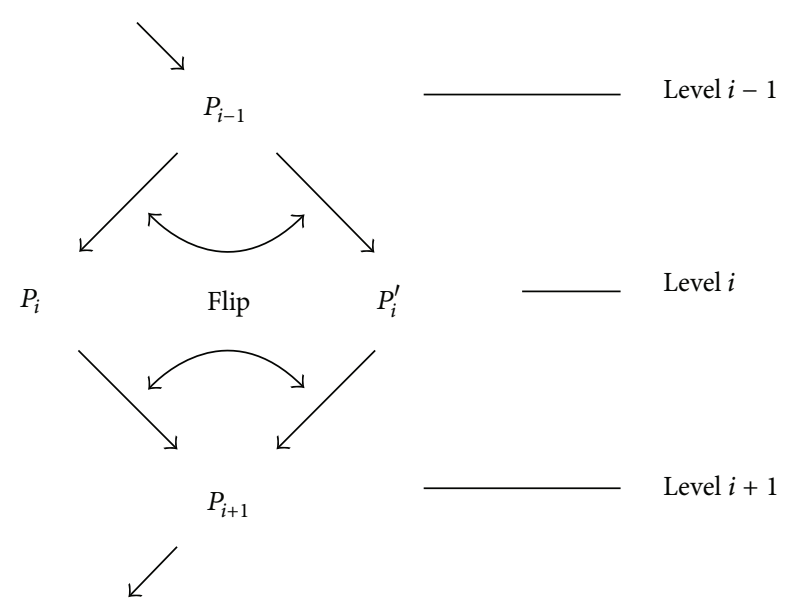

A doublet

(exactly two paths between $P_{i-1}$ and $P_{1+1}$ )

Figure 3

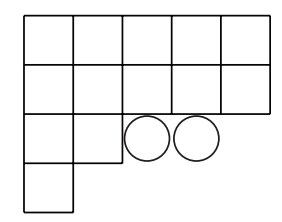

$[5521] \longrightarrow[5531] \longrightarrow[5541]$

The diagonal entry is $q$

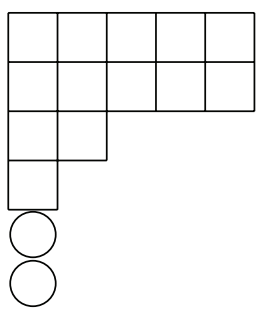

[5521] $\longrightarrow[55211] \longrightarrow[552111]$

The diagonal entry is $-1 / q$

Figure 4: Example of singlets.

the number of summations is equal to the number $\sum_{i j}\left|a_{i j}\right|$ of vertices in the braid.

\section{Examples}

To illustrate the use of these formulas, we calculate the contributions of simple diagrams $Q$ to the simple HOMFLY polynomials. There is nothing new in these formulas, they are present here just to illustrate how the method works. For numerous new examples see, [87].

If one considers only $H_{\square}$ in the fundamental representation $R=\square=$ [1], $|\square|=1$, then (20) for an $m$-strand braid involves $Q$ of the size $m$, that is, $Q$ at the level $m$ in the representation graph. To make use of (20), one needs to know the $m-1$ matrices $\widehat{\mathscr{R}}_{(i)}$.

$m=2$. There are two possible $Q=[2]$ and $Q=[11]$ and one matrix $\widehat{\mathscr{R}}_{(1)}$ in each case.

For $Q=[2]$ there is a single path from $\square=[1]$ to [2]; that is, $\bar{M}_{\square[2]}=1$; it coincides with the segment ([1], [2]); thus, according to our rules the corresponding $1 \times 1$ matrix $\widehat{\mathscr{R}}_{(1)}=q$.
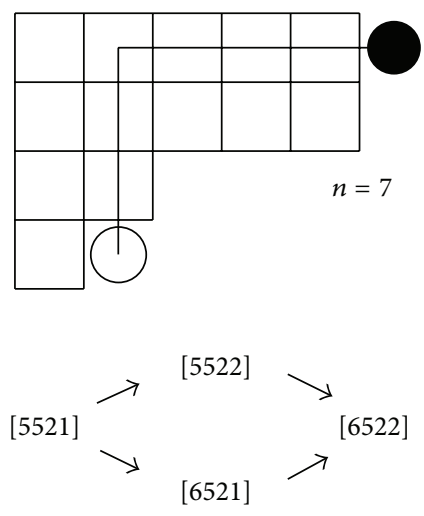

FIgURE 5: Example of doublets.

Similarly for $Q=[11]$ one gets $\widehat{\mathscr{R}}_{(1)}=-1 / q$. Thus the generic expression (19) in this case is

$$
A^{n} H_{\square}^{(n)}=q^{n} \chi_{[2]}(G)+(-q)^{-n} \chi_{[11]}(G), \quad n \text { odd. }
$$


The coefficient at the l.h.s. takes into account the normalization factor $\mathfrak{N}$.

$m=3$. For $Q=[3]$ and [111] there are unique paths from $\square$ and the corresponding matrices $\widehat{\mathscr{R}}_{(1)}=\widehat{\mathscr{R}}_{(2)}$ are again $1 \times 1$ and are equal to $q$ and $-1 / q$, respectively.

However, for $Q=[21]$ the situation is already different. There are two paths between $\square$ and [21], and the left one [1] $\rightarrow$ $[2] \rightarrow$ [21] contains the segment ([1], [2]), while the second path [1] $\rightarrow$ [11] $\rightarrow$ [21] contains the segment ([1], [11]). This means that the matrix $\widehat{\mathscr{R}}_{(1)}$ in the sector $Q=[21]$ is $2 \times 2$; it is diagonal with the entries $q$ and $-1 / q$. The matrix $\widehat{\mathscr{R}}_{(2)}$ is again $2 \times 2$, but it is not diagonal, because the two paths are connected exactly by the flip. Since the length of the hook in this case is $n=2$, our rules imply that

$$
\widehat{\mathscr{R}}_{(2)}=\left(\frac{1}{[2]_{q}}\right)\left(\begin{array}{cc}
-\frac{1}{q^{2}} & \sqrt{[3]_{q}} \\
\sqrt{[3]_{q}} & q^{2}
\end{array}\right) \text {, }
$$

and the formula for the arbitrary 3-strand braid is [85] (see Figure 6)

$$
\begin{aligned}
& A^{a_{1}+b_{1}+a_{2}+b_{2}+\cdots} H_{\square}^{\left(a_{1} b_{1}\left|a_{2} b_{2}\right| \cdots\right)} \\
& =q^{a_{1}+b_{1}+a_{2}+b_{2}+\cdots} \chi_{[3]}(G)+\left(-\frac{1}{q}\right)^{a_{1}+b_{1}+a_{2}+b_{2}+\cdots} \chi_{[111]}(G) \\
& +\operatorname{tr}_{2 \times 2}\left\{\left(\begin{array}{cc}
q & 0 \\
0 & -\frac{1}{q}
\end{array}\right)^{a_{1}}\right. \\
& \times\left(\begin{array}{cc}
-\frac{1}{q^{2}[2]_{q}} & \frac{\sqrt{[3]_{q}}}{[2]_{q}} \\
\frac{\sqrt{[3]_{q}}}{[2]_{q}} & \frac{q^{2}}{[2]_{q}}
\end{array}\right)^{b_{1}}\left(\begin{array}{cc}
q & 0 \\
0 & -\frac{1}{q}
\end{array}\right)^{a_{2}} \\
& \left.\times\left(\begin{array}{cc}
-\frac{1}{q^{2}[2]_{q}} & \frac{\sqrt{[3]_{q}}}{[2]_{q}} \\
\frac{\sqrt{[3]_{q}}}{[2]_{q}} & \frac{q^{2}}{[2]_{q}}
\end{array}\right)^{b_{2}} \cdots\right\}
\end{aligned}
$$

$m=4$. For $Q=[4]$ and [1111] all the three matrices $\widehat{\mathscr{R}}_{1,2,3}$ are $1 \times 1$ and equal to $q$ and $-1 / q$, respectively.

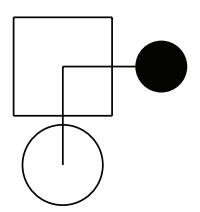

$$
\begin{gathered}
{[1] \longrightarrow[21]} \\
n=2
\end{gathered}
$$

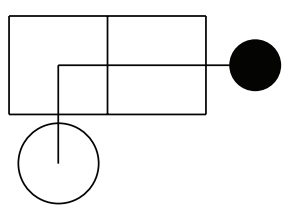

$$
\begin{gathered}
{[2] \longrightarrow[31]} \\
n=3
\end{gathered}
$$

FIgURE 6

For $Q=[31]$ there are three paths. Ordered from the left to the right they are

$$
\begin{gathered}
\alpha=[1] \longrightarrow[2] \longrightarrow[3] \longrightarrow[31], \\
\beta=[1] \longrightarrow[2] \longrightarrow[21] \longrightarrow[31], \\
\gamma=[1] \longrightarrow[11] \longrightarrow[21] \longrightarrow[31]
\end{gathered}
$$

At level 2 the flip relates $\beta$ and $\gamma$ and at level 3 relates $\alpha$ and $\beta$. This implies that in sector [31] one has [85]

$$
\begin{gathered}
\widehat{\mathscr{R}}_{(1)}=\left(\begin{array}{ccc}
q & \\
q & \\
& -\frac{1}{q}
\end{array}\right), \\
\widehat{\mathscr{R}}_{(2)}=\left(\begin{array}{ccc}
q & 0 & 0 \\
0-\frac{1}{q^{2}[2]_{q}} & \frac{\sqrt{[3]_{q}}}{[2]_{q}} \\
0 & \frac{\sqrt{[3]_{q}}}{[2]_{q}} & \frac{q^{2}}{[2]_{q}}
\end{array}\right), \\
\widehat{\mathscr{R}}_{(3)}=\left(\begin{array}{ccc}
\frac{1}{q^{3}[3]_{q}} & \frac{\sqrt{[2]_{q}[4]_{q}}}{\sqrt{[2]_{q}[4]_{q}}} & 0 \\
\frac{[3]_{q}}{0} & \frac{q^{3}}{[3]_{q}} & 0 \\
0 & 0 & q
\end{array}\right) .
\end{gathered}
$$

For $Q=[211]$ the answer is similar; the three paths are now

$$
\begin{gathered}
\bar{\alpha}=[1] \longrightarrow[2] \longrightarrow[21] \longrightarrow[211] \\
\bar{\beta}=[1] \longrightarrow[11] \longrightarrow[21] \longrightarrow[211] \\
\bar{\gamma}=[1] \longrightarrow[11] \longrightarrow[111] \longrightarrow[211]
\end{gathered}
$$




$$
\begin{gathered}
\widehat{\mathscr{R}}_{(1)}=\left(\begin{array}{ccc}
q & \\
-\frac{1}{q} & \\
& -\frac{1}{q}
\end{array}\right), \\
\widehat{\mathscr{R}}_{(2)}=\left(\begin{array}{ccc}
-\frac{1}{q^{2}[2]_{q}} & \frac{\sqrt{[3]_{q}}}{[2]_{q}} & 0 \\
\frac{\sqrt{[3]_{q}}}{[2]_{q}} & \frac{q^{2}}{[2]_{q}} & 0 \\
0 & 0 & -\frac{1}{q}
\end{array}\right), \\
\widehat{\mathscr{R}}_{(3)}=\left(\begin{array}{ccc}
-\frac{1}{q} & 0 & 0 \\
0 & -\frac{1}{q^{3}[3]_{q}} & \frac{\sqrt{[2]_{q}[4]_{q}}}{[3]_{q}} \\
0 & \frac{\sqrt{[2]_{q}[4]_{q}}}{[3]_{q}} & \frac{q^{3}}{[3]_{q}}
\end{array}\right) .
\end{gathered}
$$

For $\underline{Q=[22]}$, there are just two paths:

$$
\begin{gathered}
\delta=[1] \longrightarrow[2] \longrightarrow[21] \longrightarrow[22], \\
\bar{\delta}=[1] \longrightarrow[11] \longrightarrow[21] \longrightarrow[22]
\end{gathered}
$$

and they are related by the flip at level 2 . This means that both $\widehat{\mathscr{R}}_{(1)}$ and $\widehat{\mathscr{R}}_{(3)}$ are $2 \times 2$ and diagonal, while $\widehat{\mathscr{R}}_{(2)}$ is not:

$$
\begin{gathered}
\widehat{\mathscr{R}}_{(1)}=\widehat{\mathscr{R}}_{(3)}=\left(\begin{array}{cc}
q & 0 \\
0 & -\frac{1}{q}
\end{array}\right), \\
\widehat{\mathscr{R}}_{(2)}=\left(\begin{array}{cc}
-\frac{1}{q^{2}[2]_{q}} & \frac{\sqrt{[3]_{q}}}{[2]_{q}} \\
\frac{\sqrt{[3]_{q}}}{[2]_{q}} & \frac{q^{2}}{[2]_{q}}
\end{array}\right) .
\end{gathered}
$$

The counterpart of (10) and (12) is now obvious, but rather lengthy, so we do not write it down here.

\section{Cabling Method}

Cabling is based on the fact that the representation $R$ appears in the product of $|R|$ fundamental representations, $R \in \square^{|R|}$; thus, the answer for $H_{R}^{\mathscr{K}}$ can be extracted from the answer for $H_{\square^{\otimes|R|}}^{\mathscr{K}^{|R|}}$ by the projection:

$$
H_{R}^{\mathscr{K}}(G)=\mathfrak{N}_{c} \sum_{\mathrm{Q} \in \square^{\otimes m|R|}} \mathfrak{C}_{\square Q}^{\mathscr{K}^{|R|}} \chi_{\mathrm{Q}}(G),
$$

where the normalization factor is slightly corrected as compared with (7), see [87] for details. Cabling is widely used not only in the knot theory [101], but also in the theory of $\mathscr{R}$ matrices and integrable systems [103-106] (where it is called the fusion procedure).

Here $\mathscr{K}^{|R|}$ is an $m|R|$-strand braid, obtained from $\mathscr{K}$ by substituting each line (strand) by a bunch (cable) of $|R|$ strands so that the intersection of two strands is now a peculiar combination $\mathfrak{R}$ of $|R|^{2}$ original $\mathscr{R}$-matrices, but in the fundamental representations (see Figure 7). In other words,

$$
\mathfrak{C}_{\square Q}^{\mathscr{K}^{|R|}}=\operatorname{tr}_{M_{\square Q}} \widehat{\mathfrak{P}}_{R} \widehat{\mathfrak{R}}_{(1)}^{a_{11}} \ldots \widehat{\mathfrak{R}}_{(m-1)}^{a_{1, m-1}} \widehat{\Re}_{(1)}^{a_{21}} \ldots \widehat{\Re}_{(m-1)}^{a_{2, m-1}} \ldots
$$

$\mathfrak{P}_{R}$ is the projection from the reducible representation $\square^{\otimes|R|}$ onto $R$. It can actually be inserted everywhere in between the $\mathfrak{R}$ matrices, but for the case of knots (not links) a single insertion is sufficient.

Now, (20) can be written explicitly and calculated with the help of Section 3, but in terms of the $m|R|$-strand braid and the corresponding $\widehat{\mathscr{R}}$ matrices replaced with $\widehat{R}$. This is a rather cumbersome expression even for the simplest knots (therefore we do not rewrite (20) in this way) but absolutely straightforward and adequate for practical computations.

Moreover, here comes a bonus of the path sum representation: the projector $\mathfrak{P}_{R}$ is nearly trivial, at least in the case of knots: one should include only the directed paths from $Q$ to $\square$, which pass through the vertex $R$, this effectively decreases the size of the matrices $\widehat{\Re}_{(I)}, I=1, \ldots, m-1$ from $M_{\square \mathrm{Q}}$ to $M_{\square R} \cdot M_{R Q}$.

$N B$. The constituent matrices $\widehat{\mathscr{R}}_{(i)}, i=1, \ldots, m|R|-1$, cannot be reduced in this way: one cannot insert the projector inside $\widehat{\Re}$.

\section{Cabling for $m=2$ and $|R|=2$}

Now we can use the last example in Section 4 to demonstrate how the cabling works in our formulas. The 4-strand braids are enough to describe only the 2-strand (torus) knots in representations [2] and [11]. In this case there is just a single combined matrix:

$$
\widehat{\Re}_{(1)}=\widehat{\mathscr{R}}_{(2)} \widehat{\mathscr{R}}_{(1)} \widehat{\mathscr{R}}_{(3)} \widehat{\mathscr{R}}_{(2)} .
$$

According to our rules, if we consider, for example, $H_{[2]}^{(n)}$ for a 2 -strand knot, we should leave in $\widehat{\Re}_{(1)}$ only the paths passing through the vertex [2].

This means that the single path leading to $Q=[4]$ remains intact, while the one to [1111] is now eliminated; this means that there will be no contribution of $Q=[1111]$ to $H_{[2]}^{(n)}$. 


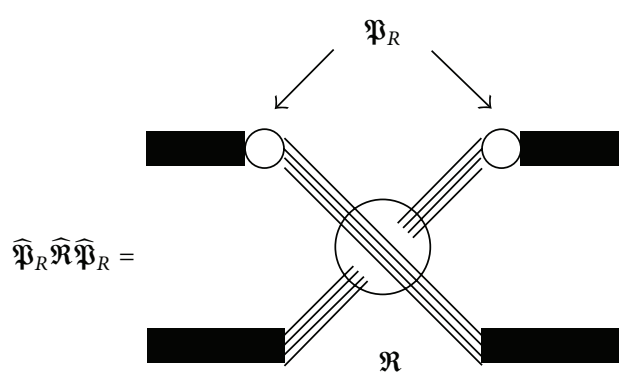

Figure 7

From the three paths which led to $Q=[31]$, only two remain, $\alpha$ and $\beta$; thus the third line and the third column should be omitted from the matrix $\widehat{\Re}$ :

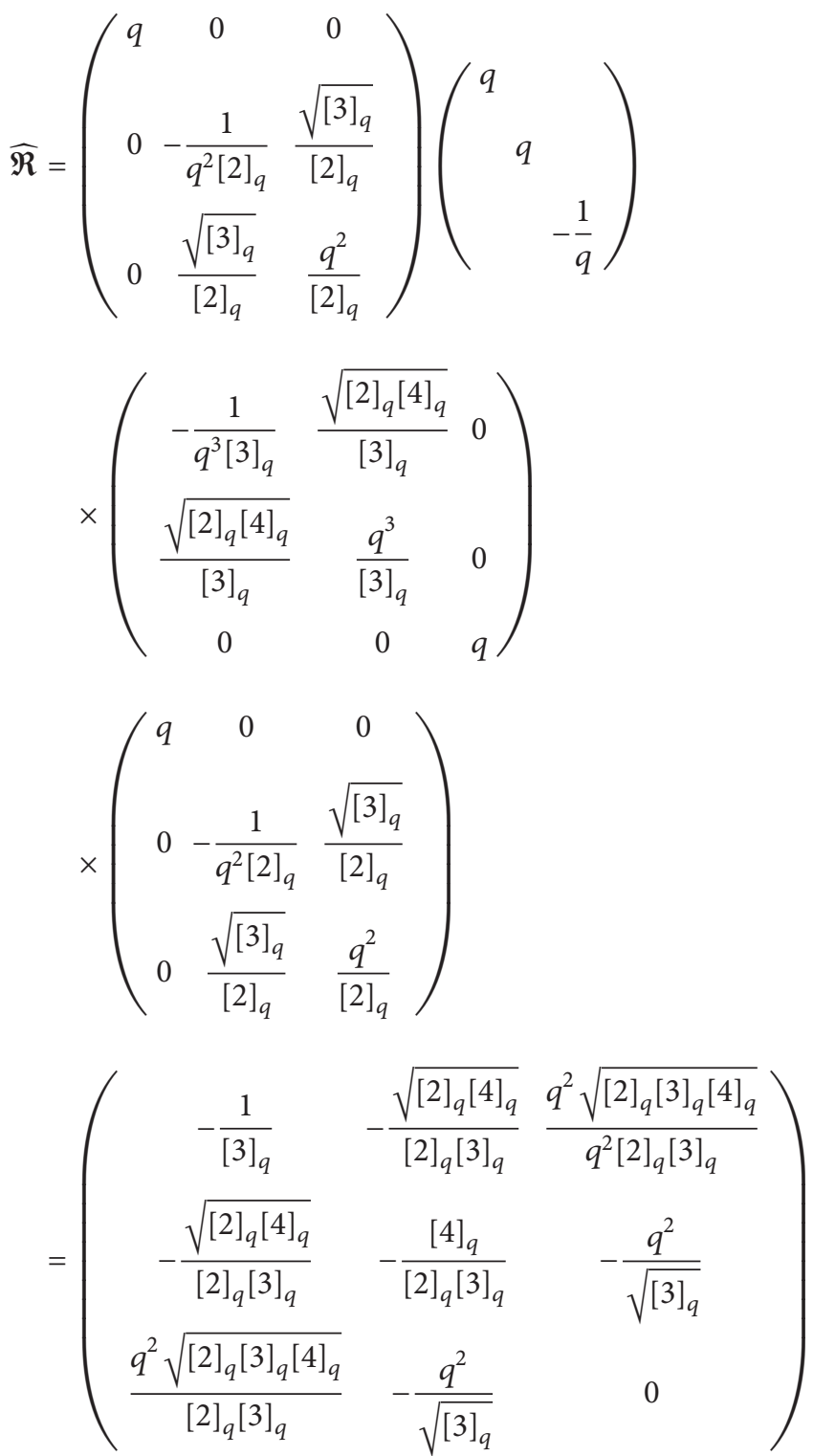

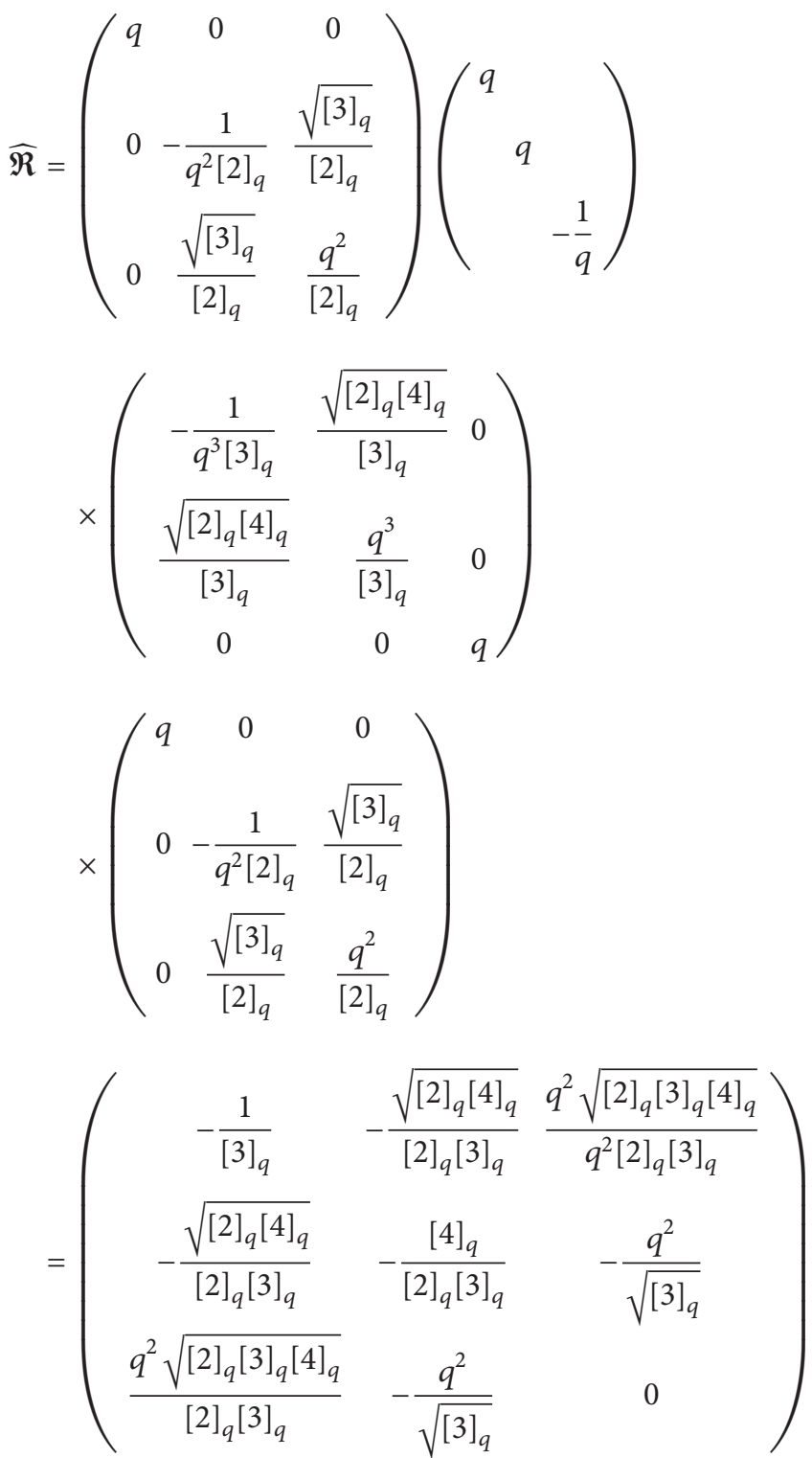

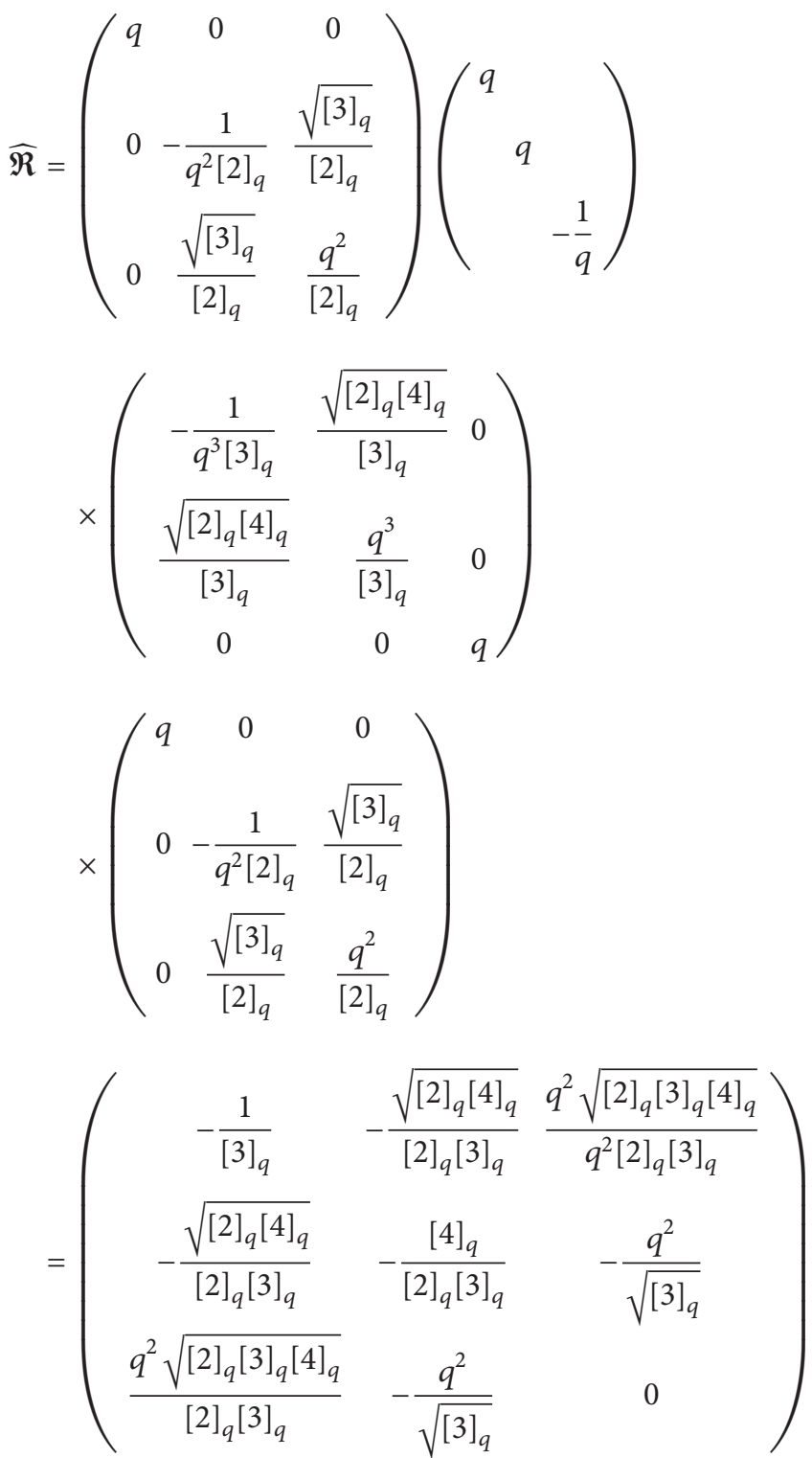

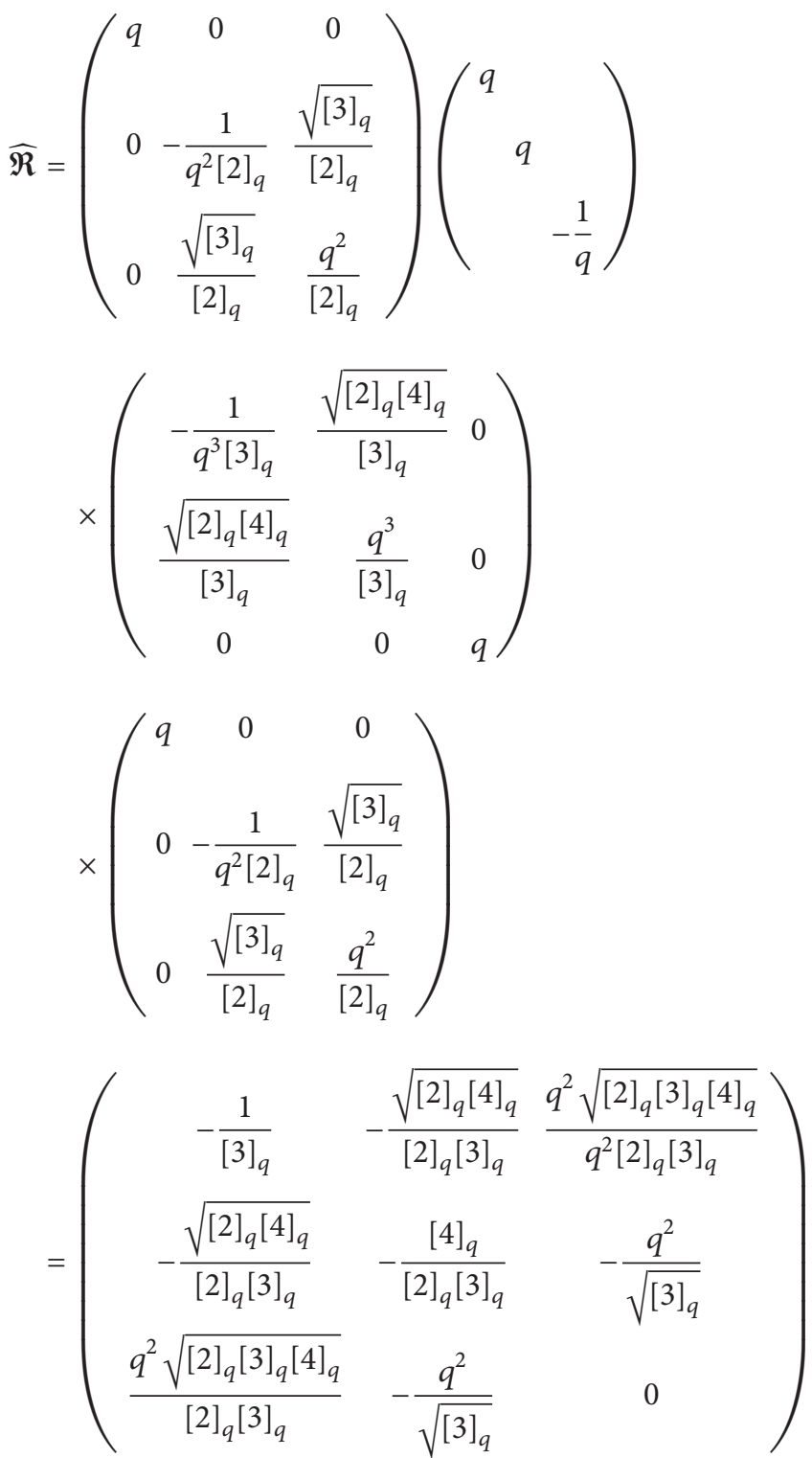

This $2 \times 2$ matrix has two eigenvalues: 0 and -1 .

Similarly from the three paths to $Q=[211]$ only one survives, $\bar{\alpha}$, and $\widehat{\Re}$ matrix is reduced to $1 \times 1$ :

$$
\widehat{\Re}=\left(\begin{array}{ccc}
-\frac{1}{q^{2}[2]_{q}} & \frac{\sqrt{[3]_{q}}}{[2]_{q}} & 0 \\
\frac{\sqrt{[3]_{q}}}{[2]_{q}} & \frac{q^{2}}{[2]_{q}} & 0 \\
0 & 0 & -\frac{1}{q}
\end{array}\right)\left(\begin{array}{rrr}
q & \\
& -\frac{1}{q} & \\
& & -\frac{1}{q}
\end{array}\right)
$$

$$
\times\left(\begin{array}{ccc}
-\frac{1}{q} & 0 & 0 \\
0 & -\frac{1}{q^{3}[3]_{q}} & \frac{\sqrt{[2]_{q}[4]_{q}}}{[3]_{q}} \\
0 & \frac{\sqrt{[2]_{q}[4]_{q}}}{[3]_{q}} & \frac{q^{3}}{[3]_{q}}
\end{array}\right)
$$$$
\times\left(\begin{array}{ccc}
-\frac{1}{q^{2}[2]_{q}} & \frac{\sqrt{[3]_{q}}}{[2]_{q}} & 0 \\
\frac{\sqrt{[3]_{q}}}{[2]_{q}} & \frac{q^{2}}{[2]_{q}} & 0 \\
0 & 0 & -\frac{1}{q}
\end{array}\right)
$$

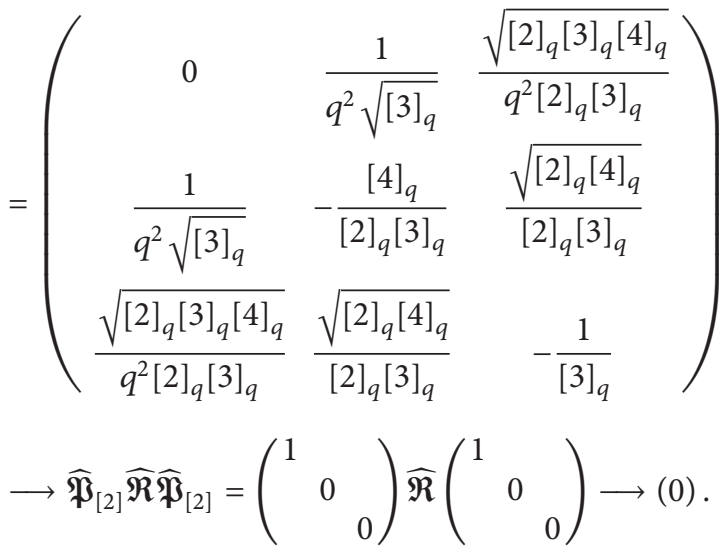


Thus, despite a path survives in the [211] sector, its contribution is actually vanishing, as it should be, because [211] $\notin$ $[2] \otimes[2]$.

Also for $Q=[22]$ only one path of the two remains, $\delta$, and

$$
\begin{aligned}
& \left(\begin{array}{cc}
-\frac{1}{q^{2}[2]_{q}} & \frac{\sqrt{[3]_{q}}}{[2]_{q}} \\
\frac{\sqrt{[3]_{q}}}{[2]_{q}} & \frac{q^{2}}{[2]_{q}}
\end{array}\right)\left(\begin{array}{cc}
q & 0 \\
0 & -\frac{1}{q}
\end{array}\right)^{2} \\
& \times\left(\begin{array}{cc}
-\frac{1}{q^{2}[2]_{q}} & \frac{\sqrt{[3]_{q}}}{[2]_{q}} \\
\frac{\sqrt{[3]_{q}}}{[2]_{q}} & \frac{q^{2}}{[2]_{q}}
\end{array}\right) \\
& =\left(\begin{array}{cc}
\frac{1}{q^{2}} & 0 \\
0 & q^{2}
\end{array}\right) \longrightarrow \widehat{\mathfrak{P}}_{[2]} \widehat{\mathfrak{R} \widehat{\mathfrak{P}}_{[2]}} \\
& =\left(\begin{array}{ll}
1 & \\
& 0
\end{array}\right) \widehat{\Re}\left(\begin{array}{ll}
1 & \\
& 0
\end{array}\right) \longrightarrow\left(\frac{1}{q^{2}}\right) .
\end{aligned}
$$

Putting everything together one gets for odd $n$ (i.e., for the knot):

$$
\begin{aligned}
& q^{2 n} A^{2 n} H_{[2]}^{(n)}=\operatorname{tr}_{[1]^{84}}^{\mathrm{grad}} \widehat{\mathfrak{P}}_{[2]} \widehat{\Re}^{n}=\operatorname{tr}_{[1]^{84}}^{\mathrm{grad}}\left(\widehat{\mathfrak{P}}_{[2]} \widehat{\Re} \widehat{\mathfrak{P}}_{[2]}\right)^{n} \\
& =q^{4 n} \chi_{[4]}(G)+\operatorname{tr}_{2 \times 2} \\
& \quad \times\left\{\left(\begin{array}{cc}
-\frac{1}{[3]_{q}} & -\frac{\sqrt{[2]_{q}[4]_{q}}}{[2]_{q}[3]_{q}} \\
-\frac{\sqrt{[2]_{q}[4]_{q}}}{[2]_{q}[3]_{q}} & -\frac{[4]_{q}}{[2]_{q}[3]_{q}}
\end{array}\right)\right\}^{n} \chi_{[31]}(G) \\
& + \\
& \quad q^{-2 n} \chi_{[22]}(G) \\
& =q^{-2 n}\left(q^{6 n} \chi_{[4]}(G)-q^{2 n} \chi_{[31]}(G)+\chi_{[22]}(G)\right) .
\end{aligned}
$$

The formula at the last line is the standard Rosso-Jones expression [107-109] for the colored HOMFLY polynomial in the case of a torus knot.

$N B$. Note that the matrices $\widehat{\Re}$ do not commute with $\widehat{\mathfrak{P}}_{R}$ and that the projector is as simple as described above only for the first cable in the braid.

Note also that two of the five $Q \in[1]^{4}$ do not contribute to the middle line for somewhat different reasons; $\chi_{[1111]}$ does not appear, because there are no paths, going from $\square$ to [1111] via [2], while for $\chi_{\text {[211] }}$ such path exists, just its contribution to the relevant element of $\widehat{\Re}$ is zero.

These examples illustrate all the peculiarities of our formulas. Once they are understood, the use of the path sum formalism is straightforward.

\section{Open Questions}

The path sum formula provides a complete solution for the evaluation problem of arbitrary colored HOMFLY polynomials. It is nice looking and theoretically attractive, and it is algorithmic and very effective for practical computations (a vast list of examples is provided in $[87,102])$. It represents the knot polynomials in the form of a character expansion $[70,85]$ and therefore expresses them directly in terms of $N$, $A$, or, the time-variables $p_{k}$, whatever one prefers. Still there are a few obvious directions to study.

First, our final formula for the colored knots heavily relies on the cabling approach and therefore is in a certain sense more involved than (7). In particular, when $R \neq \square$, the sizes of $\mathscr{R}$-matrices are considerably bigger than theoretically possible (though the entries are simpler). Of course, the path sum representation exists for the arbitrary $R$, but it is in terms of another representation graph, $\Gamma_{R}$ describing powers of the representation $R$, and the corresponding $\mathscr{R}$-matrices are more involved (their constituents are no longer just $1 \times 1$ and $2 \times 2$ blocks). At the same time that graph is the subset of the full one, $\Gamma_{\square}$, which we considered in the present letter, and the sum over the paths from $Q$ to $\square$, passing through the vertex $R$, can be also considered as a sum over just the paths from $Q$ to $R$, more in the spirit of (7). However, there are many such paths in $\Gamma_{\square}$ with the same image in $\Gamma_{R}$, and they contribute to the matrix elements of the composite $\widehat{\Re}$. The quantities (20) contain also multiple sums over the paths from $R$ to $\square$, but in fact they do not depend on this, and one can simply fix one such path arbitrarily (as a kind of a gauge fixing). It would be very interesting to find a relevant modification of the path sum formula which would not refer to the cabling procedure, and $[91,92]$ strongly implies that this can be possible and that such a formula should possess its own beauties.

Second, the representation graph exists for arbitrary Lie algebras, not only for $S U(N)$, and the sum path formula should be straightforwardly generalized to arbitrary groups, in particular, from the HOMFLY (for $S U(N)$ ) to Kauffman (for $S O(N)$ ) polynomials.

Third, it is now obvious that the Khovanov-Rozansky approach [58-63] and the superpolynomials [64, 65] should have a similar representations in terms of the multiple sums over paths (i.e., over the standard Young tableaux). There are certain advances in this direction for the torus knots [110$115]$, particularly close should be the results by $[116,117]$. We emphasize once again that the sums over Young tableaux provide the answers for arbitrary knots, not only torus; this is a theorem for the HOMFLY polynomials and a plausible conjecture for the superpolynomials.

To this list one should of course add a massive calculation of the colored HOMFLY polynomials for nontorus knots, especially in representations with many lines and rows in the Young diagram, that is, not just (anti)symmetric representations. Such examples are crucially important for understanding the structure of generic knot polynomials, for example, a la $[93-100,102,118,119]$ or $[71,72,120,121]$ or $[122]$, and various relations between them [123-127]. 


\section{Acknowledgments}

The authors are indebted to S. Artamonov, H. Itoyama, and A. Popolitov for fruitful discussions, which helped them to improve the presentation of the results from $[86,87]$ for the purposes of this paper. Thier work is partly supported by the Ministry of Education and Science of the Russian Federation under contract 8207 , by the Brazil National Counsel of Scientific and Technological Development (A. Morozov), by the Dynasty Foundation (And. Morozov), by NSh-3349.2012.2, by RFBR Grants 13-02-00457 (A. Mironov), 13-02-00478 (Al. Morozov), and 12-02-31078 mol-a (A. Anokhina; And. Morozov), by joint Grant 12-02-92108-Yaf-a, 13-02-90459Ukr-f-a, and 13-02-91371-ST-a.

\section{References}

[1] S. S. Chern and J. Simons, "Characteristic forms and geometric invariants," Annals of Mathematics, vol. 99, pp. 48-69, 1974.

[2] J. W. Alexander, "Topological invariants of knots and links," Transactions of the American Mathematical Society, vol. 30, no. 2, pp. 275-306, 1928.

[3] J. H. Conway, "Algebraic properties," in Proceedings of the AIP Conference on Computational Problems in Abstract Algebra, J. Leech, Ed., pp. 329-358, Pergamon Press, Oxford, UK, 1970.

[4] V. F. R. Jones, "Index for subfactors," Inventiones Mathematicae, vol. 72, no. 1, pp. 1-25, 1983.

[5] L. H. Kauffman, "State models and the jones polynomial," Topology, vol. 26, no. 3, pp. 395-407, 1987.

[6] P. Freyd, D. Yetter, J. Hoste, W. B. R. Lickorish, K. Millett, and A. Ocneanu, "A new polynomial invariant of knots and links," American Mathematical Society Bulletin, vol. 12, no. 2, pp. 239246, 1985.

[7] J. H. Przytycki and P. Traczyk, "Invariants of links of Conway type," Kobe Journal of Mathematics, vol. 4, no. 2, pp. 115-139, 1988.

[8] A. S. Schwarz, "New topological invariants arising in the theory of quantized fields," in Proceedings of the International Topological Conference, Part II, Baku, Azerbaijan, 1987.

[9] E. Witten, "Quantum field theory and the Jones polynomial," Communications in Mathematical Physics, vol. 121, no. 3, pp. 351399, 1989.

[10] E. Guadagnini, M. Martellini, and M. Mintchev, "Chern-Simons field theoryand quantum groups," in Quantum Groups, vol. 370, pp. 307-317, Springer, Berlin, Germany, 1990.

[11] E. Guadagnini, M. Martellini, and M. Mintchev, "Chern-Simons holonomies and the appearance of quantum groups," Physics Letters B, vol. 235, no. 3-4, pp. 275-281, 1990.

[12] N. Y. Reshetikhin and V. G. Turaev, "Ribbon graphs and their invaraints derived from quantum groups," Communications in Mathematical Physics, vol. 127, no. 1, pp. 1-26, 1990.

[13] M. Alvarez, J. M. F. Labastida, and E. Pérez, "Vassiliev invariants for links from Chern-Simons perturbation theory," Nuclear Physics B, vol. 488, no. 3, pp. 677-718, 1997.

[14] G. Moore and N. Seiberg, "Taming the conformal zoo," Physics Letters B, vol. 220, no. 3, pp. 422-430, 1989.

[15] V. V. Fock and Y. I. Kogan, "Generating function for 2+1d WZW model correlators and the Sugawara construction from the $N$ Chern-Simons theory," Modern Physics Letters A, vol. 5, no. 17, pp. 1365-1371, 1990.
[16] J. M. F. Labastida and A. V. Ramallo, "Operator formalism for Chern-Simons theories," Physics Letters B, vol. 227, no. 1, p. 92, 1989.

[17] J. M. F. Labastida and A. V. Ramallo, "Chern-Simons and conformal field theories," Nuclear Physics B, vol. 16, pp. 594-596, 1990.

[18] L. Alvarez-Gaumé, C. Gomez, and G. Sierra, "Quantum group interpretation of some conformal field theories," Physics Letters $B$, vol. 220, no. 1-2, pp. 142-152, 1989.

[19] J. Maldacena, "The large $A_{N-1}$ limit of superconformal field theories and supergravity," Advances in Theoretical and Mathematical Physics, vol. 2, no. 2, pp. 231-252, 1998.

[20] S. S. Gubser, I. R. Klebanov, and A. M. Polyakov, "Gauge theory correlators from non-critical string theory," Physics Letters B, vol. 428, no. 1-2, pp. 105-114, 1998.

[21] E. Witten, "Anti de Sitter space and holography," Advances in Theoretical and Mathematical Physics, vol. 2, no. 2, pp. 253-291, 1998.

[22] L. F. Alday, D. Gaiotto, and Y. Tachikawa, "Liouville correlation functions from four-dimensional gauge theories," Letters in Mathematical Physics A, vol. 91, no. 2, pp. 167-197, 2010.

[23] N. Wyllard, " $N=2$ conformal Toda field theory correlation functions from conformal $S U(N) N=2$ quiver gauge theories," Journal of High Energy Physics, no. 11, p. 002, 2009.

[24] A. Mironov and A. Morozov, "The power of Nekrasov functions," http://arxiv.org/abs/0908.2190.

[25] A. Mironov and A. Morozov, "On AGT relation in the case of U(3)," http://arxiv.org/abs/0908.2569.

[26] A. Mironov, S. Mironov, A. Morozov, and A. Morozov, "Resolving puzzles of massive gravity with and without violation of Lorentz symmetry," Classical and Quantum Gravity, vol. 27, no. 12, Article ID 125005, 2010.

[27] G. Bonelli and A. Tanzini, "Hitchin systems, $N=2$ gauge theories and W-gravity," http://arxiv.org/abs/0909.4031.

[28] G. Giribet, "On AGT description of $N_{f}=4$ SCFT with $N=2$," Journal of High Energy Physics, no. 1, p. 097, 18, 2010.

[29] K. Maruyoshi and M. Taki, "Deformed prepotential, quantum integrable system and Liouville field theory," http://arxiv.org/ abs/1006.4505.

[30] A. Marshakov, A. Mironov, and A. Morosov, "On AGT relations with surface operator insertion and a stationary limit of betaensembles," Journal of Geometry and Physics, vol. 61, no. 7, pp. 1203-1222, 2011.

[31] C. Kozcaz, S. Pasquetti, and N. Wyllard, "A \& B model approaches to surface operators and Toda theories," http://arxiv .org/abs/1004.2025.

[32] J.-E. Bourgine, "Large $\mathrm{N}$ techniques for Nekrasov partition functions and AGT conjecture," http://arxiv.org/abs/1212.4972.

[33] N. Seiberg and E. Witten, "Electric-magnetic duality, monopole condensation, and confinement in $N=2$ supersymmetric Yang-Mills theory," Nuclear Physics B, vol. 426, no. 1, pp. 19-52, 1994.

[34] N. Seiberg and E. Witten, "Monopoles, duality and chiral symmetry breaking in $N=2$ supersymmetric QCD," Nuclear Physics B, vol. 431, no. 3, pp. 484-550, 1994.

[35] A. Hanany and Y. Oz, "On the quantum moduli space of vacua of $N=2$ supersymmetric $N=2$ gauge theories," Nuclear Physics B, vol. 452, no. 1-2, pp. 283-312, 1995.

[36] J. Minahan and D. Nemeschansky, "Hyperelliptic curves for supersymmetric Yang-Mills," http://arxiv.org/abs/hep-th/ 9507032 . 
[37] J. Minahan and D. Nemeschansky, " $N=2$ Super Yang-Mills and Subgroups of SL(2,Z)," http://arxiv.org/abs/hep-th/9601059 .

[38] O. Aharony and S. Yankielowicz, "Exact electric-magnetic duality in $N=2$ supersymmetric QCD theories," Nuclear Physics $B$, vol. 473, no. 1-2, pp. 93-120, 1996.

[39] P. Argyres, "S-duality and global symmetries in $N=2$ supersymmetric field theory," http://arxiv.org/abs/hep-th/9706095 .

[40] P. Argyres, "Dualities in supersymmetric field theories," http:// arxiv.org/abs/hep-th/9705076.

[41] J. A. Minahan, "Duality symmetries for $N=2$ supersymmetric QCD with vanishing $R$-functions," Nuclear Physics B, vol. 537, no. 1-3, pp. 243-259, 1999.

[42] D. Galakhov, A. Mironov, and A. Morozov, "S-duality as a betadeformed Fourier transform," Journal of High Energy Physics, vol. 2012, p. 67, 2012.

[43] M. Billo, M. Frau, L. Gallot, A. Lerda, and I. Pesando, "Deformed $N=2$ theories, generalized recursion relations and S-duality," http://arxiv.org/abs/1302.0686.

[44] N. Nemkov, "S-duality as Fourier transform for arbitrary e_1,e_2," http://arxiv-web3.library.cornell.edu/abs/1307.0773.

[45] V. S. Dotsenko and V. A. Fateev, "Conformal algebra and multipoint correlation functions in 2D statistical models," Nuclear Physics B, vol. 240, no. 3, pp. 312-348, 1984.

[46] A. Marshakov, A. Mironov, and A. Morozov, "Generalized matrix models as conformal field theories. Discrete case," Physics Letters B, vol. 265, no. 1-2, pp. 99-107, 1991.

[47] S. Kharchev, A. Marshakov, A. Mironov, A. Morozov, and S. Pakuliak, "Conformal matrix models as an alternative to conventional multi-matrix models," Nuclear Physics B, vol. 404, no. 3, pp. 717-750, 1993.

[48] R. Dijkgraaf and C. Vafa, "Toda theories, matrix models, topological strings, and $N=2$ gauge systems," http://arxiv.org/ abs/0909.2453.

[49] H. Itoyama, K. Maruyoshi, and T. Oota, "The Quiver matrix model and $2 \mathrm{~d}-4 \mathrm{~d}$ conformal connection," Progress of Theoretical Physics, vol. 123, no. 6, pp. 957-987, 2010.

[50] T. Eguchi and K. Maruyoshi, "Penner type matrix model and Seiberg-Witten theory," http://arxiv.org/abs/0911.4797 .

[51] T. Eguchi and K. Maruyoshi, "Seiberg-Witten theory, matrix model and AGT relation," http://arxiv.org/abs/1006.0828 .

[52] R. Schiappa and N. Wyllard, "An A_r threesome: matrix models, 2d CFTs and $4 \mathrm{~d} N=2$ gauge theories," http://arxiv.org/abs/ 0911.5337.

[53] A. Mironov, A. Morozov, and Sh. Shakirov, "Matrix model conjecture for exact BS periods and Nekrasov functions," http://arxiv.org/abs/0911.5721 .

[54] A. Mironov, A. Morozov, and Sh. Shakirov, "Conformal blocks as Dotsenko-Fateev integral discriminants," http://arxiv .org/abs/1001.0563.

[55] A. Mironov, A. Morozov, and A. Morozov, "Conformal blocks and generalized Selberg integrals," Nuclear Physics B, vol. 843, no. 2, pp. 534-557, 2011.

[56] V. A. Alba, V. A. Fateev, A. V. Litvinov, and G. M. Tarnopolskiy, "On combinatorial expansion of the conformal blocks arising from AGT conjecture," Letters in Mathematical Physics, vol. 98, no. 1, pp. 33-64, 2011.

[57] A. Belavin and V. Belavin, "Agt conjecture and integrable structure of conformal field theory for $\mathrm{c}=1$," Nuclear Physics B, vol. 850, no. 1, pp. 199-213, 2011.

[58] M. Khovanov, "A categorification of the Jones polynomial," Duke Mathematical Journal, vol. 101, no. 3, pp. 359-426, 2000.
[59] D. Bar-Natan, “On Khovanov's categorification of the Jones polynomial," Algebraic \& Geometric Topology, vol. 2, pp. 337370, 2002.

[60] M. Khovanov and L. Rozhansky, "Matrix factorizations and link homology," http://arxiv.org/abs/math/0401268.

[61] M. Khovanov and L. Rozhansky, "Matrix factorizations and link homology II," http://arxiv.org/abs/math/0505056.

[62] N. Carqueville and D. Murfet, "Computing KhovanovRozansky homology and defect fusion," http://arxiv.org/abs/ 1108.1081 .

[63] V. Dolotin and A. Morozov, "Introduction to Khovanov homologies I. Unreduced Jones superpolynomial," Journal of High Energy Physics, no. 1, p. 065, front matter + 46, 2013.

[64] S. Gukov, A. Schwarz, and C. Vafa, "Khovanov-Rozansky homology and topological strings," Letters in Mathematical Physics, vol. 74, no. 1, pp. 53-74, 2005.

[65] N. M. Dunfield, S. Gukov, and J. Rasmussen, "The superpolynomial for knot homologies," Experimental Mathematics, vol. 15, no. 2, pp. 129-160, 2006.

[66] P. Dunin-Barkowski, A. Mironov, A. Morozov, A. Sleptsov, and A. Smirnov, "Superpolynomials for toric knots from evolution induced by cut-and-join operators," http://arxiv.org/abs/ 1106.4305.

[67] M. Aganagic and Sh. Shakirov, "Knot Homology from Refined Chern-Simons theory," http://arxiv.org/abs/1105.5117.

[68] M. Aganagic and Sh. Shakirov, "Refined Chern-Simons theory and topological string," http://arxiv.org/abs/1210.2733.

[69] A. Morozov, "Challenges of beta-deformation," http://arxiv.org/ abs/1201.4595.

[70] A. Mironov, A. Morozov, and A. Morozov, "Character expansion for HOMFLY polynomials. I. Integrability and difference equations," http://arxiv.org/abs/1112.5754.

[71] A. Mironov, A. Morozov, and A. Sleptsov, "Genus expansion of HOMFLY polynomials," http://arxiv.org/abs/1303.1015.

[72] A. Morozov, “Integrability in non-perturbative QFT"' http:// arxiv.org/abs/1303.2578.

[73] A. Mironov, A. Morozov, and S. Natanzon, "Complete set of cut-and-join operators in Hurwitz-Kontsevich theory," http:// arxiv.org/abs/0904.4227.

[74] A. Mironov, A. Morozov, and S. Natanzon, "Algebra of differential operators associated with Young diagrams," http://arxiv .org/abs/1012.0433.

[75] A. Mironov, A. Morozov, and S. Natanzon, "Integrability properties of Hurwitz partition functions. II. Multiplication of cut-and-join operators and WDVV equations," http://arxiv .org/abs/1108.0885.

[76] A. Alexandrov, A. Mironov, A. Morozov, and S. Natanzon, "Integrability of Hurwitz partition functions. I. Summary," http://arxiv.org/abs/1103.4100.

[77] A. Gerasimov, S. Khoroshkin, D. Lebedev, A. Mironov, and A. Morozov, "Generalized Hirota equations and representation theory. I. The case of SL(2) and SL_q(2)," http://arxiv.org/ abs/hep-th/9405011.

[78] S. Kharchev, A. Mironov, and A. Morozov, "Non-standard KP evolution and quantum t-function," http://arxiv.org/abs/qalg/9501013.

[79] A. Mironov, "Quantum deformations of t-functions, Bilinear identities and representation theory," http://arxiv.org/abs/hepth/9409190. 
[80] A. Mironov, "Group theory approach to the t-function and its quantization," Theoretical and Mathematical Physics, vol. 114, pp. 127-183, 1998.

[81] "Knot Atlas," Page (by D.Bar-Natan), http://katlas.org/wiki/ Main.

[82] R. K. Kaul and T. R. Govindarajan, "Three-dimensional ChernSimons theory as a theory of knots and links," Nuclear Physics $B$, vol. 380, no. 1-2, pp. 293-333, 1992.

[83] P. Ramadevi, T. R. Govindarajan, and R. K. Kaul, "Three dimensional Chern-Simons theory as a theory of knots and links III: compact semi-simple group," Nuclear Physics B, vol. 402, pp. 548-566, 1993.

[84] P. Ramadevi, T. R. Govindarajan, and R. K. Kaul, "Knot invariants from rational conformal field theories," Nuclear Physics B, vol. 422, no. 1-2, pp. 291-306, 1994.

[85] A. Mironov, A. Morozov, and A. Morozov, "Character expansion for HOMFLY polynomials. II. Fundamental representation. Up to five strands in braid," Journal of High Energy Physics, vol. 03, p. 034, 2012.

[86] A. Anokhina, A. Mironov, A. Morozov, and A. Morozov, "Racah coefficients and extended HOMFLY polynomials for all 5-, 6and 7-strand braids," Nuclear Physics B, vol. 868, no. 1, pp. 271313, 2013.

[87] A. Anokhina and An. Morozov, "Cabling procedure for the colored HOMFLY polynomials," http://arxiv-web3.library.cornell.edu/abs/1307.2216.

[88] A. Morozov and A. Smirnov, "Chern-Simons theory in the temporal gauge and knot invariants through the universal quantum A-matrix," Nuclear Physics B, vol. 835, no. 3, pp. 284-313, 2010.

[89] A. Smirnov, "Notes on Chern-Simons Theory in the Temporal Gauge," Proceedings of International School of Subnuclar Physics. Erice, Italy, 2009.

[90] D. Galakhov, A. Mironov, A. Morozov, and A. Smirnov, "On 3d extensions of AGT relation," http://arxiv.org/abs/1104.2589.

[91] H. Itoyama, A. Mironov, A. Morozov, and A. Morozov, "Character expansion for HOMFLY polynomials. III. All 3-Strand braids in the first symmetric representation," http://arxiv.org/ abs/1204.4785.

[92] H. Itoyama, A. Mironov, A. Morozov, and A. Morozov, "Eigenvalue hypothesis for Racah matrices and HOMFLY polynomials for 3-strand knots in any symmetric and antisymmetric representations," http://arxiv.org/abs/1209.6304.

[93] A. Mironov, A. Morozov, and A. Morozov, "Character expansion for HOMFLY polynomials. II. Fundamental representation. Up to five strands in braid," Journal of High Energy Physics, no. 3, p. 034, 2012.

[94] P. Ramadevi and T. Sarkar, "On link invariants and topological string amplitudes," Nuclear Physics B, vol. 600, no. 3, pp. 487511, 2001.

[95] Zodinmawia and P. Ramadevi, "SU(N) quantum Racah coefficients \& non-torus links," http://arxiv.org/abs/1107.3918.

[96] Zodinmawia and P. Ramadevi, "Reformulated invariants for non-torus knots and links," http://arxiv.org/abs/1209.1346.

[97] H. Fuji, S. Gukov, and P. Sulkowski, "Volume conjecture: refined and categorified," http://arxiv.org/abs/1203.2182.

[98] S. Nawata, P. Ramadevi, Zodinmawia, and X. Sun, "Super-Apolynomials for twist knots," http://arxiv.org/abs/1209.1409.

[99] H. Fuji, S. Gukov, M. Stosic, and P. Sulkowski, "3d analogs of Argyres-Douglas theories and knot homologies," http://arxiv .org/abs/1209.1416.
[100] S. Nawata, P. Ramadevi, and Zodinmawia, "Colored HOMFLY polynomials from Chern-Simons theory," http://arxiv.org/ abs/1302.5144.

[101] C. C. Adams, The Knot Book: An Elementary Introduction to the Mathematical Theory of Knots, W. H. Freeman and Company, New York, NY, USA, 1994.

[102] A. Anokhina, A. Mironov, A. Morozov, and An. Morozov, "Knot polynomials in the first non-symmetric representation," http://arxiv.org/abs/1211.6375.

[103] P. P. Kulish and N. Yu. Reshetikhin, "On GL3-invariant solutions to the Yang-Baxter equation and the assosiated quantum systems," Zapiski Nauchnykh Seminarov Leningradskogo Otdeleniya Matematicheskogo Instituta, vol. 120, pp. 92-121, 1982 (Russian).

[104] P. P. Kulish and N. Yu. Reshetikhin, "GL3-invariant solutions of the Yang-Baxter equation and associated quantum systems," Journal of Soviet Mathematics, vol. 34, pp. 1948-1971, 1986.

[105] P. P. Kulish and E. K. Sklyanin, "Quantum spectral transform method. Recent developments," in Integrable Quantum Field Theories, vol. 151 of Lecture Notes in Physics, pp. 61-119, Springer, Berlin, Germany, 1982.

[106] M. Jimbo, T. Miwa, and M. Okado, "Solvable lattice models whose states are dominant integral weights of Ait-1(1)," Letters in Mathematical Physics, vol. 14, no. 2, pp. 123-131, 1987.

[107] M. Rosso and V. Jones, "On the invariants of torus knots derived from quantum groups," Journal of Knot Theory and its Ramifications, vol. 2, no. 1, pp. 97-112, 1993.

[108] X.-S. Lin and H. Zheng, "On the Hecke algebras and the colored HOMFLY polynomial," Transactions of the American Mathematical Society, vol. 362, no. 1, pp. 1-18, 2010.

[109] S. Stevan, "Chern-Simons invariants of torus links," Annales Henri Poincaré, vol. 11, no. 7, pp. 1201-1224, 2010.

[110] E. Gorsky, "q,t-Catalan numbers and knot homology," http:// arxiv.org/abs/1003.0916.

[111] I. Cherednik, "Jones polynomials of torus knots via DAHA," http://arxiv.org/abs/1111.6195.

[112] A. Oblomkov, J. Rasmussen, and V. Shende, "The Hilbert scheme of a plane curve singularity and the HOMFLY homology of its link," with an Appendix by E.Gorsky, http://arxiv.org/ abs/1201.2115.

[113] A. Mironov, A. Morosov, and Sh. Shakirov, "Towards a proof of AGT conjecture by methods of matrix models," International Journal of Modern Physics A, vol. 27, no. 1, p. 1230001, 32, 2012.

[114] A. Mironov, A. Morozov, and Sh. Shakirov, "Torus HOMFLYPT as the Hall-Littlewood polynomials," Journal of Physics A, vol. 45 , no. 35, p. 11, 2012.

[115] E. Gorsky, A. Oblomkov, J. Rasmussen, and V. Shende, “Torus knots and the rational DAHA," http://arxiv.org/abs/1207.4523 .

[116] A. Negut, "Moduli of Flags of Sheaves on $\mathrm{P}^{2}$ and their K-theory," http://arxiv.org/abs/1209.4242.

[117] E. Gorsky and A. Negut, "Refined knot invariants and Hilbert schemes," http://arxiv.org/abs/1304.3328.

[118] A. Mironov, A. Morozov, and A. Morozov, "Conformal blocks and generalized Selberg integrals," Nuclear Physics B, vol. 843, no. 2, pp. 534-557, 2011.

[119] S. Arthamonov, A. Mironov, and A. Morozov, "Differential hierarchy and additional grading of knot polynomials," http:// arxiv.org/abs/1306.5682.

[120] A. Morozov, "Special colored superpolynomials and their representation-dependence," Journal of High Energy Physics, no. 12, p. 116, 2012. 
[121] A. Morozov, "The first-order deviation of superpolynomial in an arbitrary representation from the special polynomial ", http://arxiv.org/abs/1211.4596.

[122] A. Brini, B. Eynard, and M. Mariño, "Torus knots and mirror symmetry," Annales Henri Poincaré, vol. 13, no. 8, pp. 1873-1910, 2012.

[123] R. Gelca, "Non-commutative trigonometry and the A-polynomial of the trefoil knot," Mathematical Proceedings of the Cambridge Philosophical Society, vol. 133, no. 2, pp. 311-323, 2002.

[124] R. Gelca and J. Sain, "The noncommutative A-ideal of a (2, 2p + 1)-torus knot determines its Jones polynomial," Journal of Knot Theory and its Ramifications, vol. 12, no. 2, pp. 187-201, 2003.

[125] S. Gukov, "Three-dimensional quantum gravity, Chern-Simons theory, and the A-polynomial," Communications in Mathematical Physics, vol. 255, no. 3, pp. 577-627, 2005.

[126] S. Garoufalidis, "On the characteristic and deformation varieties of a knot," in Proceedings of the Casson Fest, vol. 7 of Geometry \& Topology Monographs, pp. 291-309, Geometry \& Topology, 2004.

[127] A. Mironov and A. Morozov, "Equations on knot polynomials and 3d/5d duality," AIP Conference Proceedings, vol. 1483, pp. 189-211, 2012. 

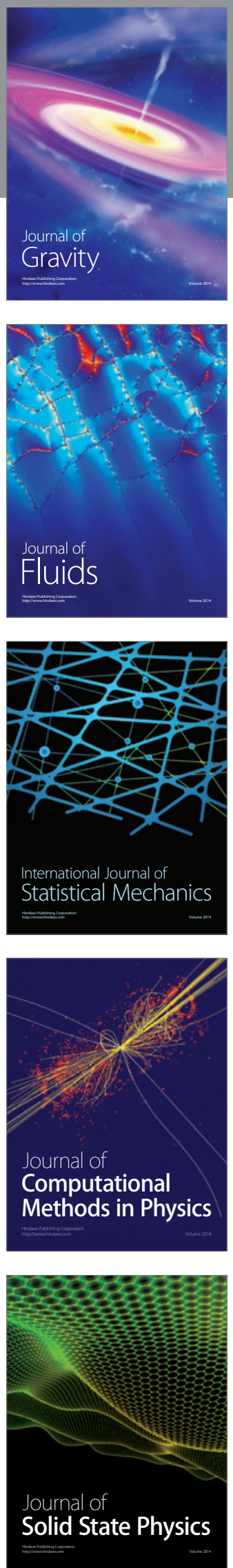

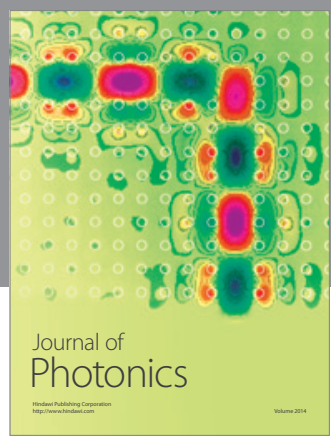

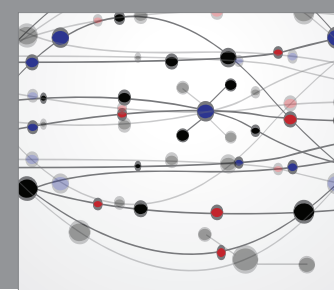

The Scientific World Journal

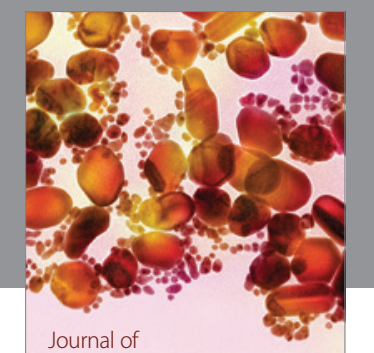

Soft Matter
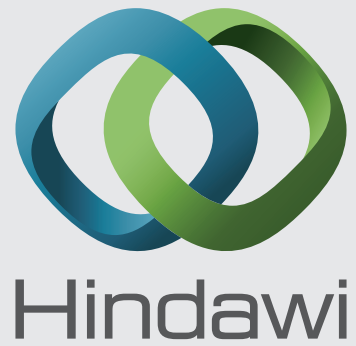

Submit your manuscripts at

http://www.hindawi.com
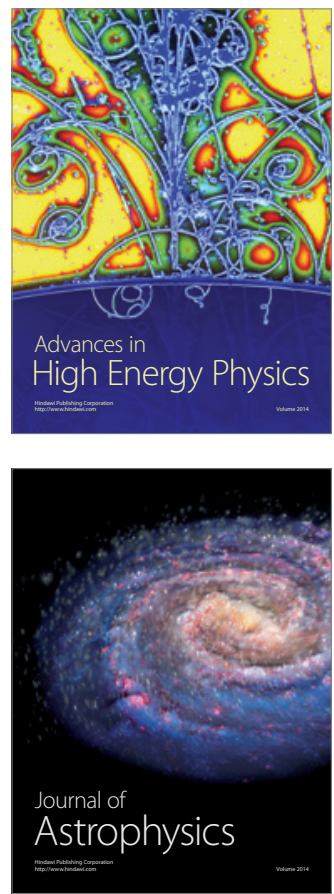
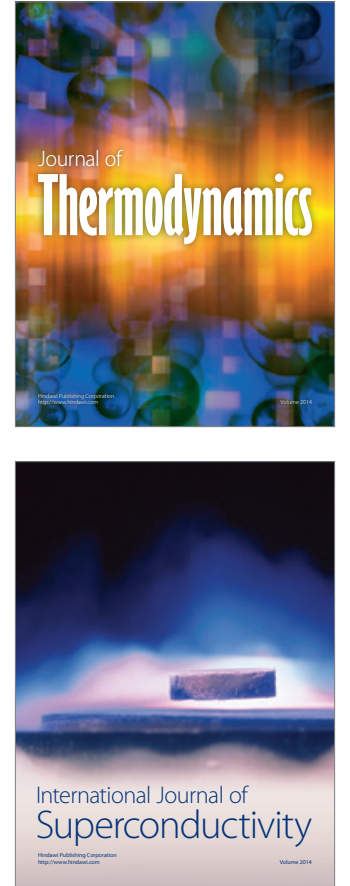
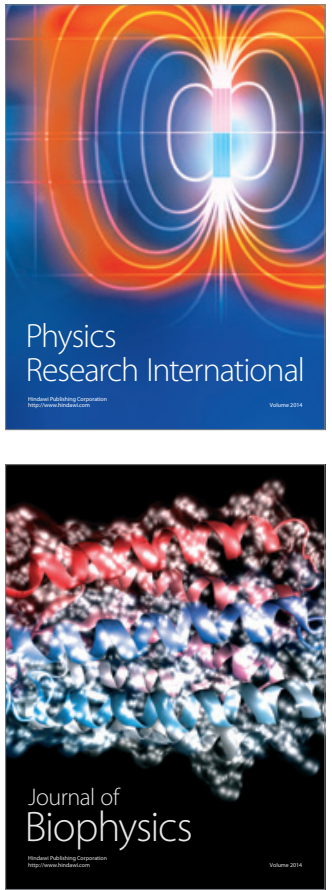
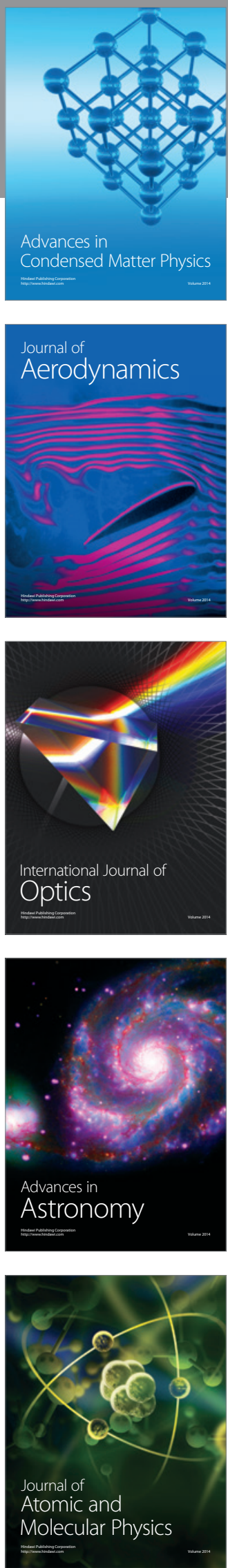